\title{
A safe transition to a more personalized alignment in total knee arthroplasty: the importance of a "safe zone" concept
}

\author{
Rüdiger von Eisenhart-Rothe ${ }^{1} \cdot$ Sebastien Lustig ${ }^{2} \cdot$ Heiko Graichen $^{3} \cdot$ Peter P. Koch ${ }^{4} \cdot$ Roland Becker $^{5}$. Arun Mullaji ${ }^{6}$. \\ Michael T. Hirschmann ${ }^{7,8}$
}

Received: 21 November 2021 / Accepted: 6 December 2021 / Published online: 20 January 2022

(c) The Author(s) under exclusive licence to European Society of Sports Traumatology, Knee Surgery, Arthroscopy (ESSKA) 2021

Medicine has been under a constant change. The latest strong and permanent trend in most of the medical fields is personalized medicine. Personalized medicine or theragnostics means targeting your diagnostics and treatment more towards the individual patient than to the mean of your patient population. In contrast, historical and current knee arthroplasty alignment and implantation concepts followed and still follow a systematic approach, mostly the mechanical alignment concept $[14,15,17]$. However, the wind of change has yet arrived in total knee arthroplasty. If one would have asked knee arthroplasty surgeons about their preferred alignment concept following ten years ago, it predominantly would have been mechanical alignment. The most personalized alignment concept in the past was

Michael T. Hirschmann

michael.hirschmann@unibas.ch

1 Department of Orthopedics and Sports Orthopedics, Klinikum Rechts Der Isar, Technical University Munich, Ismaningerstr. 22, 81675 München, Germany

2 Orthopaedic Surgery and Sports Medicine Department, Croix-Rousse Hospital, Lyon University Hospital, Lyon, France

3 Department of Arthroplasty, Sports Medicine and Traumatology, Orthopaedic Hospital Lindenlohe, Lindenlohe 18, 92421 Schwandorf, Germany

4 Gelenkzentrum Winterthur AG, CH-8400 Winterthur, Switzerland

5 Centre of Orthopaedics and Traumatology, Brandenburg Medical School Theodor Fontane, Hochstrasse 29, 14770 Brandenburg an der Havel, Germany

6 Consultant Orthopaedic Surgeon, Breach Candy Hospital, and Mullaji Knee Clinic, Bhulabhai Desai Road, Mumbai 400036, India

7 University of Basel, Basel, Switzerland

8 Department of Orthopaedic Surgery and Traumatology, Kantonsspital Baselland (Bruderholz, Liestal, Laufen), CH-4101 Bruderholz, Switzerland anatomical alignment, which still is a systematic approach and leads to a neutral mechanical alignment, however, puts the tibial joint line in $3^{\circ}$ varus and the femoral one in $3^{\circ}$ valgus.

The paramount base of every personalization is big data, which needs to be generated and collected in a standardized manner. Hence, it is no surprise that the first steps of personalized medicine in knee arthroplasty came along with the introduction of navigation, patient-specific instrumentation (PSI) and more recently robotic-assisted TKA [5, 18]. For all these systems radiological or morphometric data were collected pre- and/or intraoperatively in a large manner. Recently, in some landmark studies such data have been used to characterize the individual knee shape, morphometry and alignment phenotypes [7, 8, 14, 15]. Based on this knowledge a variety of alignment concepts were introduced. The most important ones are the adjusted mechanical alignment, the kinematic alignment, the restricted kinematic alignment, the inverse restricted kinematic alignment, the phenotype alignment and the functional alignment $[1-4,7-11,16,17$, 19]. What do you do? What should everyone do?

Nowadays, there are such a variety of different alignment concepts that even knee arthroplasty experts struggle to understand and are not always able to clearly differentiate each concept from the other. New alignment concepts from innovating surgeons appear and claim to become the new gold standard, however, with still limited data available, but more personal or expert opinions. There is a considerable number of pertinent open questions. Are the different alignment concepts leading to the same results in all patients? Are these concepts safe in all patients? What is the limit of each alignment concept and how do we improve to achieve the aimed alignment? Can we safely and reliably differentiate between constitutional and degenerative deformities? And is the specific prosthesis design suitable for this specific individual alignment? 
One feels like being in the years of an "alignment" gold rush. However, after the gold rush often there are the years of depression, may be related to unexpected complications, such as early loosening or patellofemoral maltracking. But how can we prevent the depression from happening?

A clear concept of safely shifting from mechanical alignment to a more personalized alignment in TKA is needed. Why? To reduce the number of unhappy patients. For the sake of our patients, we need to define safe zones of tibial and femoral component positioning for different knee phenotypes. These should include the hip-knee-ankle angle, the lateral distal femoral angle, the medial proximal tibial angle, the joint line obliquity and the femoral rotation $[7,8]$. To the coronal phenotype concept should be added data of sagittal and rotational alignment.

Moreover, the envelope of laxity of each knee should also be analysed and considered.

Traditionally all gaps are considered to be equal, but maybe we should aim for a more anatomical approach. Several studies have shown that the flexion gap is larger than the extension gap and the lateral flexion gap is larger than the medial flexion gap [6]. Interestingly, medial gaps in flexion and extension were all equal.

This issue of KSSTA strives to shed some light on the issue of safe zones in TKA. It includes a systematic review dealing with currently safe positions of tibial and femoral components in TKA and defining safe zones for the time of transition $[12,13]$. Further articles highlight the surgical consequences of different alignment techniques in neutral, varus and valgus phenotypes. In addition, these articles highlight the fact that the discussion about novel alignment techniques should be done based on knee phenotypes. In neutral knees, even when considering variable joint lines, the differences between different alignment techniques are not as huge as in most varus or valgus knees. A fact which should make us rethink our scientific approach towards clinical outcomes in TKA. It is highly recommended to focus on outcome studies categorized by different knee phenotypes. By including this kind of information outcomes of different alignment philosophies can be compared with each other and we can identify those knees in which one workflow is performing better than another. Finally, what about the design of our implants? Do we need to adapt the design of the current available implants to our new philosophies?

To answer those relevant questions data from registries might also be needed as huge samples are required.

Furthermore, comparison of different alignment concepts should always include a quality control between aimed and achieved alignment.

\section{References}

1. Blakeney W, Clement J, Desmeules F, Hagemeister N, Riviere C, Vendittoli PA (2019) Kinematic alignment in total knee arthroplasty better reproduces normal gait than mechanical alignment. Knee Surg Sports Traumatol Arthrosc 27(5):1410 1417. https://doi.org/10.1007/s00167-018-5174-1

2. Bonnin MP, Beckers L, Leon A, Chauveau J, Muller JH, Tibesku CO, Ait-Si-Selmi T (2020) Custom total knee arthroplasty facilitates restoration of constitutional coronal alignment. Knee Surg Sports Traumatol Arthrosc. https://doi.org/10.1007/ s00167-020-06153-8

3. Calliess T, Ettinger M, Savov P, Karkosch R, Windhagen $\mathrm{H}$ (2018) Individualized alignment in total knee arthroplasty using image-based robotic assistance: Video article. Orthopade 47(10):871-879. https://doi.org/10.1007/s00132-018-3637-1

4. Chang JS, Kayani B, Wallace C, Haddad FS (2021) Functional alignment achieves soft-tissue balance in total knee arthroplasty as measured with quantitative sensor-guided technology. J Bone Jt Surg 103(3):507-514. https://doi.org/10.1302/0301-620X. 103B.BJJ-2020-0940.R1

5. Czurda T, Fennema P, Baumgartner M, Ritschl P (2010) The association between component malalignment and post-operative pain following navigation-assisted total knee arthroplasty: results of a cohort/nested case-control study. Knee Surg Sports Traumatol Arthrosc 18(7):863-869. https://doi.org/10.1007/ s00167-009-0990-y

6. Graichen H, Lekkreusuwan K, Eller K, Grau T, Hirschmann MT, Scior W (2021) A single type of varus knee does not exist: morphotyping and gap analysis in varus OA. Knee Surg Sports Traumatol Arthrosc. https://doi.org/10.1007/ s00167-021-06688-4

7. Hirschmann MT, Moser LB, Amsler F, Behrend H, Leclercq V, Hess S (2019) Phenotyping the knee in young non-osteoarthritic knees shows a wide distribution of femoral and tibial coronal alignment. Knee Surg Sports Traumatol Arthrosc 27(5):13851393. https://doi.org/10.1007/s00167-019-05508-0

8. Hirschmann MT, Moser LB, Amsler F, Behrend H, Leclerq V, Hess S (2019) Functional knee phenotypes: a novel classification for phenotyping the coronal lower limb alignment based on the native alignment in young non-osteoarthritic patients. Knee Surg Sports Traumatol Arthrosc 27(5):1394-1402. https://doi. org/10.1007/s00167-019-05509-z

9. Howell SM, Howell SJ, Kuznik KT, Cohen J, Hull ML (2013) Does a kinematically aligned total knee arthroplasty restore function without failure regardless of alignment category? Clin Orthop Relat Res 471(3):1000-1007. https://doi.org/10.1007/ s11999-012-2613-z

10. Lustig S, Sappey-Marinier E, Fary C, Servien E, Parratte S, Batailler C (2021) Personalized alignment in total knee arthroplasty: current concepts. SICOT J 7:19. https://doi.org/10.1051/ sicotj/2021021

11. Malavolta M, Compagnoni R, Mezzari S, Calanna F, Pastrone A, Randelli P (2020) Good clinical results using a modified kinematic alignment technique with a cruciate sacrificing medially stabilised total knee arthroplasty. Knee Surg Sports Traumatol Arthrosc. https://doi.org/10.1007/s00167-020-06196-x

12. Mullaji A, Bhoskar R, Singh A, Haidermota M (2021) Knee Surg Sports Traumatol Arthrosc. https://doi.org/10.1007/ s00167-021-06796-1

13. Mullaji A, Shah R, Bhoskar R, Singh A, Haidermota M, Thakur $H$ (2021) Valgus arthritic knees can be classified into nine 
phenotypes. Knee Surg Sports Traumatol Arthrosc. https://doi. org/10.1007/s00167-021-06676-8

14. Oussedik S, Abdel MP, Victor J, Pagnano MW, Haddad FS (2020) Alignment in total knee arthroplasty. Bone Jt J 102(3):276-279. https://doi.org/10.1302/0301-620X.102B3.BJJ-2019-1729

15. Parratte S, Pagnano MW, Trousdale RT, Berry DJ (2010) Effect of postoperative mechanical axis alignment on the fifteen-year survival of modern, cemented total knee replacements. J Bone Jt Surg Am 92(12):2143-2149. https://doi.org/10.2106/JBJS.I.01398

16. Riviere C, Harman C, Boughton O, Cobb J (2020) The Kinematic Alignment Technique for Total Knee Arthroplasty. In: Riviere C, Vendittoli PA (eds) Personalized Hip and Knee Joint Replacement. Cham (CH), pp 175-195. doi:https://doi.org/10.1007/9783-030-24243-5_16

17. Riviere C, Iranpour F, Auvinet E, Howell S, Vendittoli PA, Cobb J, Parratte S (2017) Alignment options for total knee arthroplasty: a systematic review. Orthop Traumatol Surg Res 103(7):10471056. https://doi.org/10.1016/j.otsr.2017.07.010
18. Schotanus MGM, Thijs E, Heijmans M, Vos R, Kort NP (2018) Favourable alignment outcomes with MRI-based patient-specific instruments in total knee arthroplasty. Knee Surg Sports Traumatol Arthrosc 26(9):2659-2668. https://doi.org/10.1007/ s00167-017-4637-0

19. Winnock de Grave P, Luyckx T, Claeys K, Tampere T, Kellens J, Muller J, Gunst P (2020) Higher satisfaction after total knee arthroplasty using restricted inverse kinematic alignment compared to adjusted mechanical alignment. Knee Surg Sports Traumatol Arthrosc. https://doi.org/10.1007/s00167-020-06165-4

Publisher's Note Springer Nature remains neutral with regard to jurisdictional claims in published maps and institutional affiliations. 